\title{
Probing compositeness with Higgs Boson decays at the LHC
}

\author{
Maria Hoffmann ${ }^{1, a}$, Anna Kaminska ${ }^{2}$, Rosy Nicolaidou ${ }^{1}$, Stathes Paganis ${ }^{3,4}$ \\ ${ }^{1}$ DSM/IRFU (Institut de Recherches sur les Lois Fondamentales de l'Univers), CEA Saclay (Commissariat a l'Energie Atomique), 91191 \\ Gif-sur-Yvette, France \\ ${ }^{2}$ Deutsches Elektronen-Synchrotron DESY, 22607 Hamburg, Germany \\ ${ }^{3}$ Department of Physics, National Taiwan University, No. 1, Sec. 4, Roosevelt Road, 10617 Taipei, Taiwan \\ ${ }^{4}$ Department of Physics and Astronomy, The University of Sheffield, Sheffield S3 7RH, UK
}

Received: 31 July 2014 / Accepted: 12 November 2014 / Published online: 26 November 2014

(C) The Author(s) 2014. This article is published with open access at Springerlink.com

\begin{abstract}
A method is proposed to directly probe the Higgs boson compositeness using the unique characteristics of a boosted Higgs boson produced in association with a weak gauge boson $\left(W^{ \pm}, Z\right)$. The discovery potential for the upcoming LHC running is presented, showing that compositeness scales up to $3 \mathrm{TeV}$ can be probed at the LHC with an integrated luminosity of $\mathcal{L}=3000 \mathrm{fb}^{-1}$ collected at $\sqrt{s}=13 \mathrm{TeV}$.
\end{abstract}

\section{Introduction}

After the discovery of a particle consistent with the standard model (SM) Higgs boson [1-3] by the ATLAS and CMS Collaborations $[4,5]$, intense research for the understanding of the details of the Higgs mechanism has commenced. Experimental data does not rule out a possible composite nature of the Higgs boson. In composite Higgs models, the Higgs boson is a (pseudo-) Goldstone boson emerging as a result of spontaneously broken global symmetry of a new, strong dynamics. Like in QCD, there is a new fermion sector causing spin-1 $\rho$-like bound states with masses at the compositeness energy scale.

The $\rho$-like bound states $\left(\rho^{0}, \rho^{+}, \rho^{-}\right)$couple to SM particles and can hence be directly probed at the LHC through searches for single lepton events $\left(\rho^{ \pm} \rightarrow \ell^{ \pm} v\right)$ and searches for resonances decaying to two $\left(\rho^{0} \rightarrow \ell^{+} \ell^{-}\right)$and three charged leptons $\left(\rho^{ \pm} \rightarrow W^{ \pm} Z \rightarrow \ell^{ \pm} v \ell^{+} \ell^{-}\right)$.

Currently, the dilepton searches set the best limits on compositeness [6,7]. However, for a large part of the compositeness model phase space, the branching ratio (BR) of $\rho^{0} \rightarrow \ell^{+} \ell^{-}$falls very fast with the $\rho$ mass. Meanwhile, the BR of $\rho \rightarrow V H$, where $\mathrm{V}=\mathrm{W}^{ \pm}, \mathrm{Z}$, reaches a maximum. An observation of $\mathrm{VH}$ events in excess of what is expected

\footnotetext{
a e-mail: maria.hoffmann@cern.ch
}

by the SM and with an invariant mass at the $\mathrm{TeV}$ scale would be strong evidence for compositeness.

The decay products originating from heavy $\rho$ decays are characterized by a very high transverse momentum $\left(p_{\perp}\right)$. By exploiting this feature we propose a method to explore the presence of compositeness via the search for Higgs bosons with high $p_{\perp}$. This search is complementary to current searches using the dilepton invariant mass, and it may be more powerful than the latter in the parts of the compositeness model phase space where the $\operatorname{BR}\left(\rho^{0} \rightarrow \ell^{+} \ell^{-}\right) \lesssim 0.5 \%$.

In this work we search for Higgs boson decays in the two channels providing the experimentally cleanest signatures for reconstructing the Higgs; the "golden" decay to four leptons, $H \rightarrow Z Z^{*} \rightarrow 4 \ell$, where $\ell=e, \mu$, and the decay to two photons, $H \rightarrow \gamma \gamma$. The addition of the $H \rightarrow b \bar{b}$ mode is also discussed.

Since a Higgs originating from heavy $\rho$ decays will carry a large $p_{\perp}$, all non-SM backgrounds for $H \rightarrow Z Z^{*} \rightarrow 4 \ell$ and $H \rightarrow \gamma \gamma$ decays are expected to be small and SM Higgs backgrounds will thus dominate. In the $H \rightarrow b \bar{b}$ channel the non-Higgs background is still significant. The SM backgrounds can be strongly suppressed with a high Higgs $p_{\perp}$ requirement, which is the approach employed in this work. With the proposed method we are able to set a direct limit on the existence of compositeness and its energy scale.

\section{Spin-1 resonances as a signal of composite Higgs}

In this section we examine theories where the Higgs boson is a composite pseudo-Nambu-Goldstone boson (PGB) [8]. In these theories, a new strongly interacting sector with global symmetry $\mathcal{G}$ is present at the $\gtrsim 1 \mathrm{TeV}$ compositeness scale $(f)$. A composite Higgs boson emerges, much like the pion of QCD, as the PGB of a global symmetry breaking $\mathcal{G} \rightarrow \mathcal{H}$ of that sector. The explicit symmetry breaking is induced by 


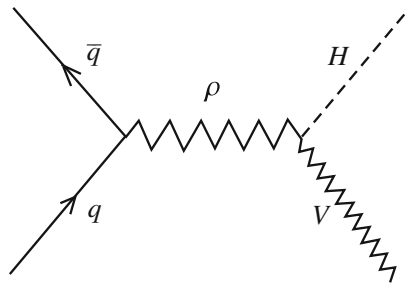

Fig. 1 Diagram depicting the process $p p \rightarrow \rho \rightarrow V H$

interactions of the SM gauge bosons and fermions with the strong sector.

The simplest example of such a strong sector is $S O(5) \rightarrow$ $S O(4)$ [8], where $S O(4) \sim S U(2)_{L} \times S U(2)_{R}$, and the $10-6=4$ pseudo-Goldstones form a complex scalar field $S U(2)_{L}$ doublet that plays the role of the Higgs. The $S O(4) \sim S U(2)_{L} \times S U(2)_{R}$ global symmetry is gauged by the electroweak symmetry $\mathcal{G}_{\mathrm{SM}}=S U(2)_{L} \times U(1)_{Y}$ of the SM, which is external to the new strong sector. This means that the SM gauge bosons are external and couple to the strong sector. Interactions of the SM gauge bosons and fermions with the strong sector are responsible for the explicit breaking of the global symmetry $\mathcal{G}$. In this picture, loops of SM fermions and gauge bosons generate a Higgs potential which eventually breaks electroweak symmetry at scale $v$. This dynamically generated electroweak scale $v$ may be lower than the strong sector (compositeness) breaking scale $f$. The ratio between the two scales $\xi=(v / f)^{2}$ is determined by the orientation of the electroweak group $\mathcal{G}_{\mathrm{SM}}$ with respect to the unbroken strong sector group $\mathcal{H}=S U(2)_{L} \times S U(2)_{R}$ in the true vacuum. If these two groups are misaligned, the electroweak symmetry is broken. Three composite pseudoGoldstones become the longitudinal degrees of freedom of the weak gauge bosons and the fourth PGB defined along the misalignment angle $\theta$ is the light Higgs boson (Fig. 1).

In such composite Higgs models, vector meson $\rho$-like resonances appear and mix with the gauge bosons building new spin-1 eigenstates. Fermion resonances from the strong sector mix with SM fermions allowing them to interact directly with $\rho$ resonances.

In this paper we consider the simplest case of the Minimal Composite Higgs Model (MCHM) [8]. The phenomenology of $\rho$ resonances in this model, transforming in the adjoint representation of $S U(2)_{L}$, is representative of the entire family of composite Higgs models. It is expected that $S U(2)_{L} \times S U(2)_{R} \subset \mathcal{H}$ for any $\mathcal{G} \rightarrow \mathcal{H}$ composite Higgs model, in order to have custodial symmetry embedded in the construction. Since vector meson resonances appear in representations of the unbroken global group $\mathcal{H}, \rho$ resonances transforming as $(3,1)$ of $S U(2)_{L} \times S U(2)_{R}$ are a generic prediction of theories with a composite Higgs boson. Such resonances are expected to have substantial interactions with SM particles due to their natural mixing with $W_{\mu}$ fields. Gen- eral properties of $S U(2)_{L} \rho$ mesons are well described in the framework of MCHM.

In the following analysis we consider $\rho$ resonances transforming as a triplet of $S U(2)_{L}$, generically expected in composite Higgs models as discussed above. For the effective description of spin-1 resonances we follow the CCWZ approach $[9,10]$, just as is done in [11] and presented in the appendix. This approach is fully equivalent to the Hidden Local Symmetries formalism [12]. Hence it is compatible with any generic composite Higgs construction used in the literature on the level of effective Lagrangian description. Moreover, it has a direct connection with deconstruction of extra dimensions, as discussed in [13].

When mixing effects in the spin-1/2 sector are neglected, compositeness models with a single vector meson can be described by just three parameters: the $\rho$ mass, $m_{\rho}$, the $\rho$ self-coupling $g_{\rho}$, and the parameter $\xi$. Based on naive dimensional analysis (NDA), the mass scale of $\rho$ resonances is expected to be $m_{\rho} \sim g_{\rho} f$, where $1<g_{\rho}<4 \pi$. The $\rho$ production cross section is dominated by the Drell-Yan process. Neutral $\rho$ decays to dileptons are particularly appealing to experimental searches for compositeness at the LHC. In particular for small $m_{\rho}(<2 \mathrm{TeV})$ and small values of parameter $\xi(\lesssim 0.1)$ the BR of $\rho^{0} \rightarrow \ell^{+} \ell^{-}$is large. However, observation of an excess in this channel would not by itself be sufficient to claim the observation of a composite $\rho$. Observation of the rest of the modes shown in Fig. 2 would be required.

Electroweak precision observables (see for example the discussion in [14]) and LHC collider data are sensitive to compositeness and can set limits in parts of the parameter space. Significant enhancement in the $H \rightarrow Z \gamma$ yield can also be induced by compositeness effects [16]. ATLAS and CMS have set limits on the $\xi$ parameter using Higgs couplings $\xi<0.22$ at $95 \% \mathrm{CL}$, restricting the compositeness scale to $f>0.5 \mathrm{TeV}[17,18]$. It is safe to say that $\xi=0.1$ at this point is still consistent with both electroweak precision constraints and LHC Higgs data, hence it will be used as a benchmark value in this paper. The compositeness scale can also be probed by direct searches for lightest vector resonances, though there is no strict relation between $m_{\rho}$ and $f$. However, as mentioned before, by NDA we can expect $m_{\rho} \sim g_{\rho} f$. Using this assumption, the LHC narrow mass dilepton searches place limits in the region of $f \lesssim 1.5$ $\mathrm{TeV} / g_{\rho}[6,7]$. These searches are most efficient for exploring the light $\rho$ resonance $\left(m_{\rho} \lesssim 2 \mathrm{TeV}\right)$ parameter space with substantial branching ratios into lepton pairs. However, for a big part of the parameter space the dilepton BR drops quickly to zero as a function of $m_{\rho}$, while the BR of $\rho \rightarrow V H$ tends to a maximum of about $70 \%$. The $\rho$ BRs as a function of the $m_{\rho}$ are shown in Fig. 2 for the benchmark parameter values of $\xi=0.1$ and $g_{\rho}=4$. Naively one would expect the BRs into VV and into VH to be equal. However, the fact 

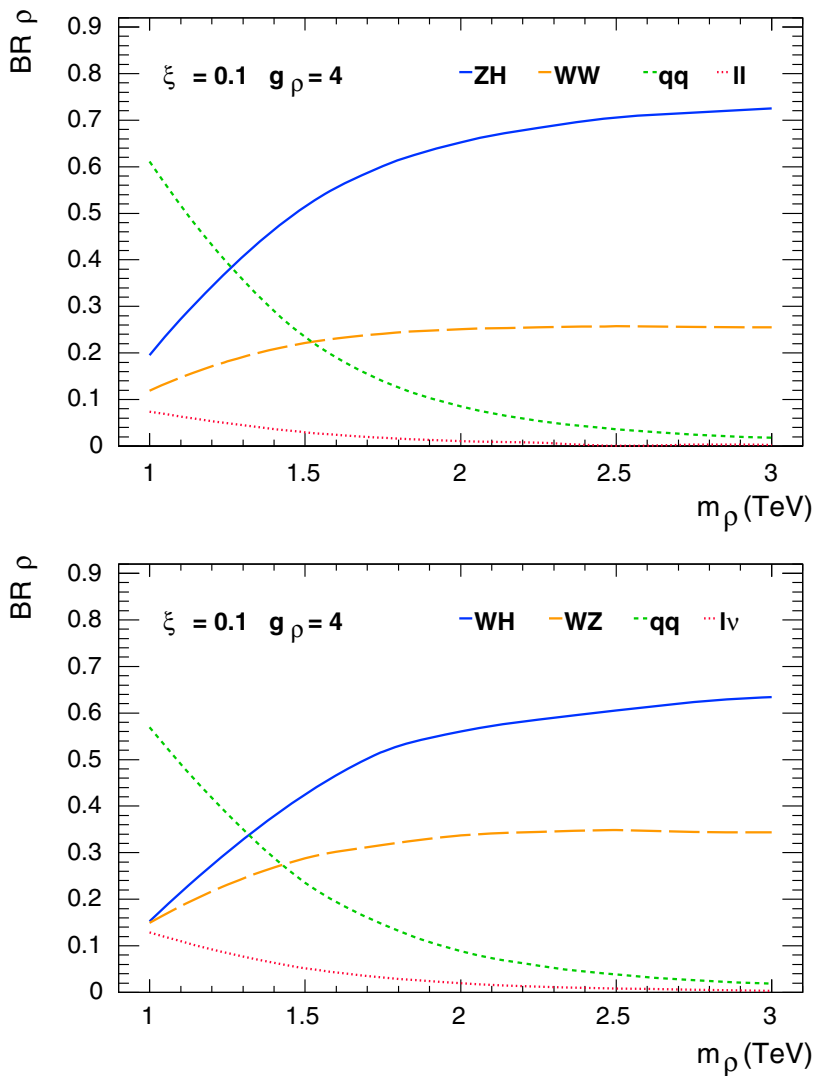

Fig. 2 Branching ratios of $\rho^{0}$ (top) and $\rho^{ \pm}$(bottom) decay modes for the benchmark parameters $\xi=0.1$ and $g_{\rho}=4$. The dilepton BR drops quickly to zero as a function of $m_{\rho}$ while the BR of $\rho \rightarrow V H$ tends to a maximum of about $70 \%$

that the cosine of the Weinberg angle $\cos \theta_{W}$ is not equal 1 leads to substantial differences in the $\rho$ couplings to $W$ and $Z$ bosons in some parts of the parameter space (especially for $\left.g_{\rho} \lesssim 6\right)$. For $m_{\rho}>2 \mathrm{TeV}$, the $\operatorname{BR}\left(\rho^{0} \rightarrow \ell^{+} \ell^{-}\right)$drops to values below $1 \%$. The branching ratios of $\rho$ mesons do not depend strongly on the choice of $g_{\rho}$, but the production cross section behaves roughly as $1 / g_{\rho}^{2}$, hence exclusion limits for $\rho$ are $g_{\rho}$ dependent. In general it is expected that $g_{\rho}$ is substantially larger than the weak couplings $g, g^{\prime}$. The $\rho$ meson of QCD is described by $g_{\rho} \sim 6$. In this paper we consider $g_{\rho}=4$ as a benchmark value, which is sufficiently above the weak couplings scale but still allows for significant $\rho$ production at the LHC.

The large expected $\mathrm{VH}$ branching ratio of the $\rho$ meson and the fact that both charged and neutral $\rho$ 's are involved offers a new possibility in experimental searches. We propose to search for boosted Higgs bosons produced by $\rho^{0}, \rho^{+}, \rho^{-}$decays in association with gauge bosons. For $\operatorname{BR}\left(\rho^{0} \rightarrow \ell^{+} \ell^{-}\right) \gtrsim 0.5 \%$ the proposed search is complementary to the dilepton search and can add information on the origin of a potential excess seen in the dilepton mass spectrum. For smaller $\mathrm{BR}\left(\rho^{0} \rightarrow \ell^{+} \ell^{-}\right)$, the $\mathrm{VH}$ search becomes the most powerful in exploring the compositeness parame- ter space. In addition, a salient feature of the VH decay is that the Higgs invariant mass may be used to suppress the background.

\section{Monte Carlo samples}

The results presented in this paper are based on Monte Carlo (MC) samples generated with MadGraph5 [23] and parton showered with PYTHIA [24]. To simulate the response of an LHC-like experiment, realistic resolution and reconstruction efficiencies for electrons, muons, photons and jets were applied with the Delphes framework [25].

The signal samples include the processes $p p \rightarrow \rho \rightarrow$ $V H$, where the definitions from Sect. 1 apply. These samples were generated at $\sqrt{s}=13 \mathrm{TeV}$ with the benchmark parameter values $\xi=0.1$ and $g_{\rho}=4$. Typical values for the $\rho$ production cross section as a function of the $\rho$ mass computed with MadGraph5 are presented in Table 1. Numerical values of the BR for the processes $\rho^{0} \rightarrow Z H, \rho^{0} \rightarrow \ell^{+} \ell^{-}$, $\rho^{ \pm} \rightarrow W^{ \pm} H$, and $\rho^{ \pm} \rightarrow \ell^{ \pm} v$ are shown in Table 2 .

The Higgs boson in the signal samples decays via either of the two channels $H \rightarrow \gamma \gamma$ or $H \rightarrow Z Z^{*} \rightarrow 4 \ell$, where $\ell=e, \mu$. The vector boson produced in association with

Table 1 Total and individual cross sections for the process $p p \rightarrow \rho$ as a function of $m_{\rho}$. The cross sections were computed at $\sqrt{s}=13 \mathrm{TeV}$ with the parameter values $\xi=\left(v / \Lambda_{c}\right)^{2}=0.1, g_{\rho}=4$. The statistical uncertainty is less than $10^{-7} \mathrm{fb}$, and therefore not quoted in this table

\begin{tabular}{lcccc}
\hline $\mathrm{m}_{\rho}[\mathrm{TeV}]$ & $\sigma_{\rho^{0}}[\mathrm{fb}]$ & $\sigma_{\rho^{+}}[\mathrm{fb}]$ & $\sigma_{\rho^{-}}[\mathrm{fb}]$ & Total [fb] \\
\hline 1.50 & 59.11 & 92.27 & 32.94 & 184.3 \\
1.75 & 27.92 & 45.25 & 14.91 & 88.08 \\
2.00 & 13.94 & 23.35 & 7.172 & 44.46 \\
2.25 & 7.245 & 12.48 & 3.606 & 23.33 \\
2.50 & 3.873 & 6.835 & 1.874 & 12.58 \\
2.75 & 2.121 & 3.807 & 0.992 & 6.920 \\
3.00 & 1.118 & 2.144 & 0.543 & 3.805 \\
\hline
\end{tabular}

Table 2 Branching ratios for the decays $\rho^{0} \rightarrow Z H \rightarrow \ell^{+} \ell^{-} H, \rho^{ \pm} \rightarrow$ $W^{ \pm} H$, and $\rho^{ \pm} \rightarrow \ell^{ \pm} v$ as a function of $m_{\rho}$. The branching ratios were computed with the parameter values $\xi=\left(v / \Lambda_{c}\right)^{2}=0.1, g_{\rho}=4$

\begin{tabular}{lllll}
\hline $\mathrm{m}_{\rho}[\mathrm{TeV}]$ & $\rho^{0} \rightarrow Z H$ & $\rho^{0} \rightarrow \ell^{+} \ell^{-}$ & $\rho^{ \pm} \rightarrow W^{ \pm} H$ & $\rho^{ \pm} \rightarrow \ell^{ \pm} v$ \\
\hline 1.50 & 0.515 & 0.0297 & 0.416 & 0.0716 \\
1.75 & 0.603 & 0.0179 & 0.499 & 0.0442 \\
2.00 & 0.653 & 0.0110 & 0.551 & 0.0277 \\
2.25 & 0.683 & 0.00703 & 0.584 & 0.0180 \\
2.50 & 0.704 & 0.00469 & 0.604 & 0.0120 \\
2.75 & 0.714 & 0.00316 & 0.617 & 0.00833 \\
3.00 & 0.725 & 0.00229 & 0.627 & 0.00593 \\
\hline
\end{tabular}


the Higgs boson is constrained to decay hadronically, i.e. $V \rightarrow j j$.

As already mentioned, the main source of background in this search is SM Higgs production. In this study we consider the three main Higgs production mechanisms at the LHC; gluon-gluon fusion ( $\mathrm{ggF})$, vector boson fusion (VBF) and associated production $(\mathrm{WH} / \mathrm{ZH}) .{ }^{1}$ All samples were generated with a Higgs mass of $m_{H}=125 \mathrm{GeV}$, and scaled with the relevant cross sections and branching ratios reported by the LHC Higgs cross-section working group and the Particle Data Group [15,28].

In the following we further assess the improvement of the search when adding the high BR channel $H \rightarrow b \bar{b}$. This is done by extrapolating the results obtained for the $H \rightarrow \gamma \gamma$ channel using results from a recent combination reported by the LHC experiments in [21,22].

\section{Analysis strategy}

The analysis method presented in this paper aims toward optimizing the discovery potential of a composite Higgs during the upcoming LHC runs. A discovery could come in the form of a direct observation of Higgs boson events with anomalously high $p_{\perp}$ or $\rho$ decays to $\mathrm{VH}$ along with an excess of events with dilepton invariant mass at the $\mathrm{TeV}$ scale.

The distribution of the transverse momentum of SM Higgs bosons and of Higgs bosons produced from $\rho$ decays is shown in Fig. 3. Given the noticeable difference in the shape of the distributions, the transverse momentum can be used as a discriminating variable to suppress the SM background. The large transverse boost of these Higgs events causes the opening angle $\Delta R=\sqrt{\Delta \eta^{2}+\Delta \phi^{2}}$ between the decay products $(Z Z$ and $\gamma \gamma)$ to be significantly smaller than that from a SM Higgs, which is shown in Fig. 4. The characteristic $\Delta R$ is not exploited in the present analysis strategy, but may be used in future searches to increase the sensitivity.

\subsection{Event selection}

A simple event selection inspired by the analysis strategies of ATLAS and CMS is implemented. The $H \rightarrow \gamma \gamma$ signal is selected by requiring two hard photons with $E_{T}$ of the leading (subleading) photon being $\geq 40$ (30) GeV. Events from the $H \rightarrow Z Z^{*} \rightarrow 4 \ell$ decay are selected by requiring two pairs of oppositely charged, same-flavor leptons. The three leptons in the quadruplet with the largest transverse momentum must, in descending order, satisfy $p_{\perp} \geq 20,15$, $10 \mathrm{GeV}$. Muons, electrons and photons must, respectively,

\footnotetext{
1 The cross section of a Higgs boson produced in association with a pair of top or b quarks $(t \bar{t} H$ or $b \bar{b} H)$ is $\approx 3$ times lower than the WH/ZH production at $\sqrt{s}=13 \mathrm{TeV}$ and therefore not considered in this study.
}

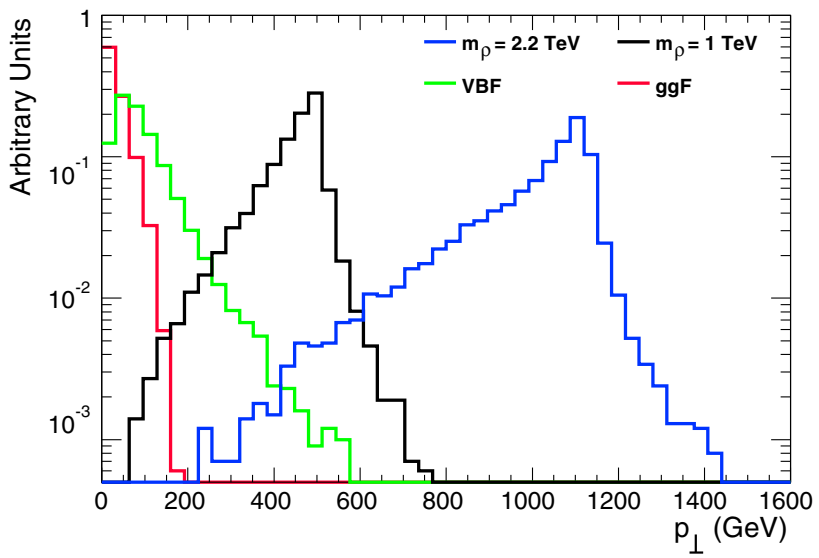

Fig. 3 The distribution of transverse momentum $p_{\perp}$ of SM Higgs bosons and of Higgs bosons originating from the $\rho \rightarrow V H$ decay

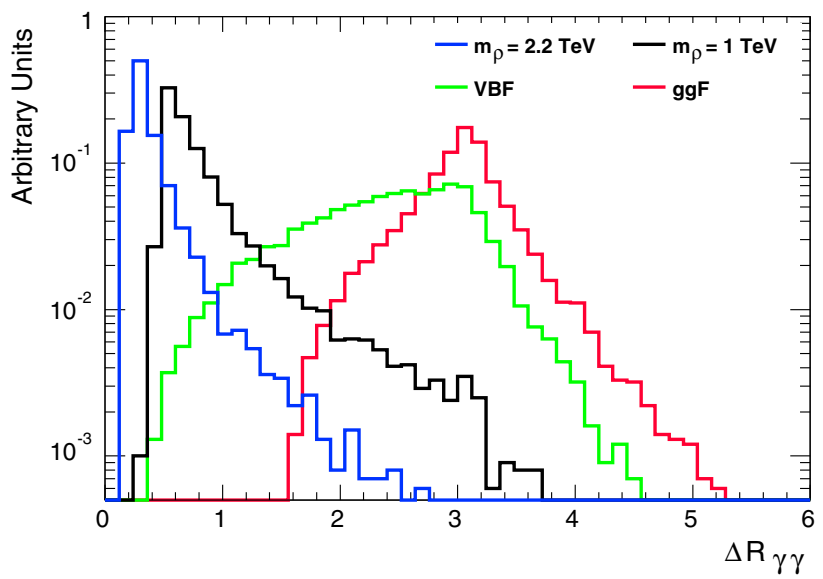

Fig. 4 The opening angle $\Delta R=\sqrt{\Delta \eta^{2}+\Delta \phi^{2}}$ between two photons from SM Higgs boson decays and from $\rho \rightarrow V H \rightarrow V \gamma \gamma$ decays

satisfy $|\eta|<2.7,2.47,2.37$. Common for the channels is that the invariant mass of the Higgs final states ${ }^{2}$ must be in the range $100-150 \mathrm{GeV}$. To suppress the SM backgrounds, an additional requirement on the transverse momentum of the Higgs system of $p_{\perp} \geq 550 \mathrm{GeV}$ is applied.

Since the applied event selection is simplified with respect to that of the LHC experiments, all samples are multiplied with analysis efficiencies representative of those presented by ATLAS and CMS. These values were obtained at $\sqrt{s}=8$ $\mathrm{TeV}[19,20]$, and have been scaled to $\sqrt{s}=13 \mathrm{TeV}$ by assuming a slight increase in efficiency. A factor of 0.4 is applied to the signal samples in both Higgs decay channels. The factor applied to the $H \rightarrow Z Z^{*} \rightarrow 4 \ell(H \rightarrow \gamma \gamma)$ background sample is $0.3(0.4)$ for $g g F, 0.2(0.5)$ for $\mathrm{VBF}$, and $0.5(0.5)$ for $\mathrm{WH} / \mathrm{ZH}$. Furthermore, the signal is multiplied by a scale factor accounting for the increased detector fiducial acceptance, which is a consequence of the boost that causes the Higgs to be emitted at lower $\eta$. The scale factor applied

\footnotetext{
2 i.e. The $4 \ell$ and $\gamma \gamma$ system.
} 
Table 3 Number of background events remaining after the full selection in Sect. 4.1 with an integrated luminosity of $\mathcal{L}=1 \mathrm{fb}^{-1}$ at $\sqrt{s}=13$ $\mathrm{TeV}$

\begin{tabular}{lll}
\hline SM production & $H \rightarrow Z Z^{*} \rightarrow 4 \ell$ & $H \rightarrow \gamma \gamma$ \\
\hline $\mathrm{ggF}$ & 0 & 0 \\
$\mathrm{VBF}$ & $1.1 \times 10^{-4}$ & $5.2 \times 10^{-3}$ \\
$\mathrm{ZH}$ & $4.0 \times 10^{-5}$ & $5.6 \times 10^{-4}$ \\
$\mathrm{WH}$ & $6.3 \times 10^{-5}$ & $8.8 \times 10^{-4}$ \\
\hline
\end{tabular}

Table 4 Number of signal events remaining after the full selection in Sect. 4.1 with an integrated luminosity of $\mathcal{L}=1 \mathrm{fb}^{-1}$ at $\sqrt{s}=13 \mathrm{TeV}$

\begin{tabular}{lll}
\hline $\mathrm{m}_{\rho}[\mathrm{TeV}]$ & $H \rightarrow Z Z^{*} \rightarrow 4 \ell$ & $H \rightarrow \gamma \gamma$ \\
\hline 1.50 & $3.8 \times 10^{-3}$ & $6.0 \times 10^{-2}$ \\
1.75 & $2.6 \times 10^{-3}$ & $3.9 \times 10^{-2}$ \\
2.00 & $1.5 \times 10^{-3}$ & $2.3 \times 10^{-2}$ \\
2.25 & $8.9 \times 10^{-4}$ & $1.5 \times 10^{-2}$ \\
2.50 & $5.1 \times 10^{-4}$ & $8.3 \times 10^{-3}$ \\
2.75 & $2.9 \times 10^{-4}$ & $4.7 \times 10^{-3}$ \\
3.00 & $1.6 \times 10^{-4}$ & $2.6 \times 10^{-3}$ \\
\hline
\end{tabular}

to the $H \rightarrow \gamma \gamma$ channel is 1.2 , while 1.3 is applied to $H \rightarrow$ $Z Z^{*} \rightarrow 4 \ell$.

The contribution from the non-resonant QCD background in the $\gamma \gamma+j j$ final state is estimated by extrapolating the obtained number of background events reported in [29] with the expected increase in production cross section [30], multiplied with the selection efficiency of the $p_{\perp}$ requirement. This efficiency is estimated with a $p p \rightarrow \gamma \gamma+j j$ sample generated with MadGraph and found to be on the order of $10^{-4}$. With an integrated luminosity of $3,000 \mathrm{fb}^{-1}$ the number of expected events from this process is more than one order of magnitude smaller than what is expected from the dominant SM Higgs background. This particular background is therefore considered safe to ignore. Similarly, the contribution from the $Z Z$ continuum ( $p p \rightarrow Z Z$ ) is assumed to be negligible after the rather tight $p_{\perp}$ requirement.

The numbers of signal and background events per $\mathrm{fb}^{-1}$ remaining after the full selection are presented in Tables 3 and 4 . As seen, the contribution from ggF production is completely suppressed.

\section{Results: discovery potential at the LHC}

The analysis described in Sect. 4 is used to assess the compositeness discovery potential at the LHC. The results are presented in terms of significance defined as $Z=$ $\sqrt{2[(s+b) \ln (1+s / b)-s]}$ [26], with $s$ and $b$ being the number of signal and background events remaining after the full selection.

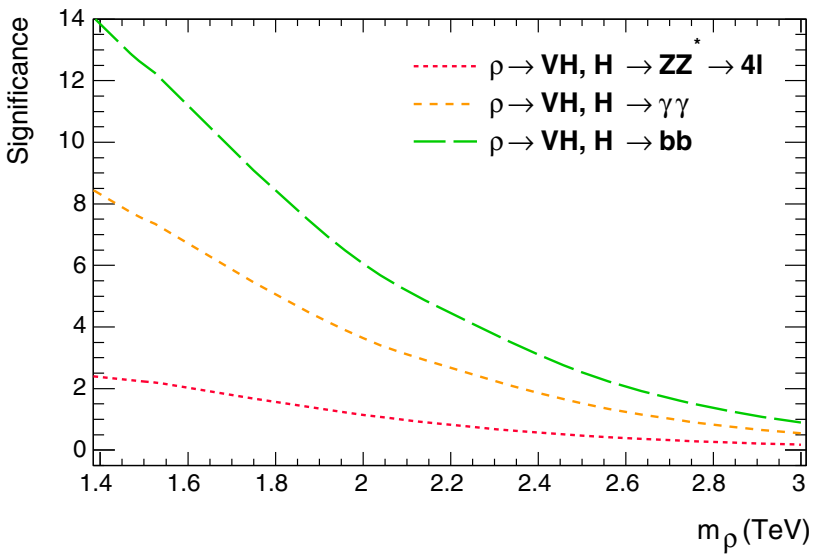

Fig. 5 Expected significance as a function of $m_{\rho}$ obtainable with a single LHC experiment using the $H \rightarrow \gamma \gamma, H \rightarrow Z Z^{*} \rightarrow 4 \ell$, and $H \rightarrow b \bar{b}$ decay channels. The latter was obtained with an optimistic extrapolation of the results obtained with the $H \rightarrow \gamma \gamma$ channel with the results reported in $[21,22]$. The significance was computed with an integrated luminosity of $\mathcal{L}=300 \mathrm{fb}^{-1}$ collected at $\sqrt{s}=13 \mathrm{TeV}$ with the parameter values $\xi=\left(v / \Lambda_{c}\right)^{2}=0.1, g_{\rho}=4$

The expected impact of the inclusion of the $H \rightarrow b \bar{b}$ channel is also considered.The problem with this channel is the presence of significant QCD non-SM Higgs background requiring careful subtraction and detailed treatment of systematics. A realistic estimate of the impact of $H \rightarrow b \bar{b}$ can be obtained using existing analyses in similar event topologies to the VH. Recent studies at the LHC [21,22] showed that when searching for a Higgs boson produced via the $t \bar{t} H$ production mechanism, the expected significance increases by a factor of 1.7 when combining the $H \rightarrow \gamma \gamma$ channel with $H \rightarrow b \bar{b}$. The result reported in $[21,22]$ is based on a detailed, full simulation including all systematics. Therefore, to realistically include the impact of the $H \rightarrow b \bar{b}$ channel, the significance obtained here with the $H \rightarrow \gamma \gamma$ channel is extrapolated by the factor 1.7 .

The significance as a function of the $\rho$ mass scale $\left(m_{\rho}\right)$ is considered for two integrated luminosity points: $\mathcal{L}=300$ $\mathrm{fb}^{-1}$ and $\mathcal{L}=3000 \mathrm{fb}^{-1}$. These sample sizes correspond to the expected integrated luminosity recorded after LHC and HL-LHC operation anticipated around the years 2020 and 2030, respectively [27]. The results obtained for the individual channels at $\sqrt{s}=13 \mathrm{TeV}$ are presented in Figs. 5 and 6 for $m_{\rho}$ in the interval $1.4-3 \mathrm{TeV}$.

As observed in Figs. 5 and 6 the diphoton channel is more sensitive than the four-lepton channel. This feature is a result of the $\operatorname{BR}(H \rightarrow \gamma \gamma)$ being more than an order of magnitude larger than $\operatorname{BR}\left(H \rightarrow Z Z^{*} \rightarrow 4 \ell\right)$. The drop in significance with increasing $m_{\rho}$ is a result of the $\rho$ production cross section decreasing with higher $m_{\rho}$. From Fig. 5 we see that a single LHC experiment with a data sample of $\mathcal{L}=300$ $\mathrm{fb}^{-1}$ is able to observe a signal with a significance of $3 \sigma$ at $m_{\rho} \sim 2.5 \mathrm{TeV}$. With $\mathcal{L}=3000 \mathrm{fb}^{-1}$ the search is sensi- 


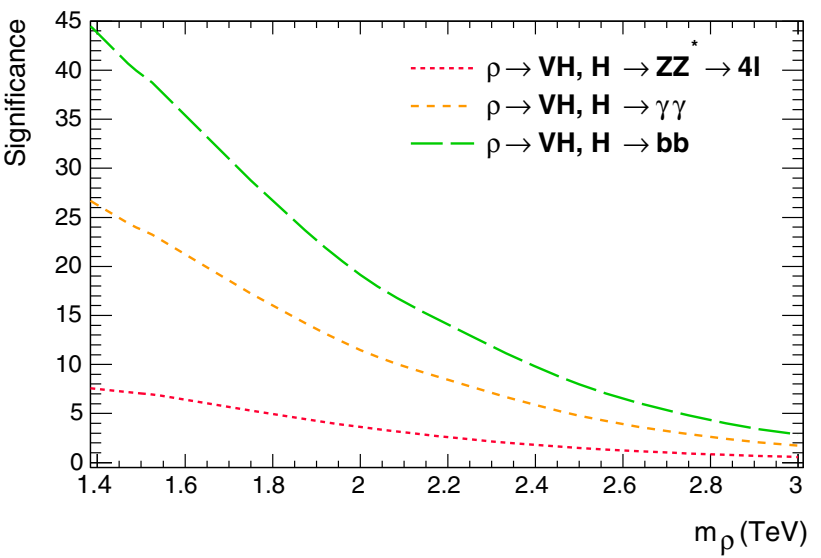

Fig. 6 Expected significance as a function of $m_{\rho}$ obtainable with a single LHC experiment using the $H \rightarrow \gamma \gamma, H \rightarrow Z Z^{*} \rightarrow 4 \ell$, and $H \rightarrow b \bar{b}$ decay channels. The latter was obtained with an optimistic extrapolation of the results obtained with the $H \rightarrow \gamma \gamma$ channel with the results reported in [21,22]. The significance was computed with an integrated luminosity of $\mathcal{L}=3000 \mathrm{fb}^{-1}$ collected at $\sqrt{s}=13 \mathrm{TeV}$ with the parameter values $\xi=\left(v / \Lambda_{c}\right)^{2}=0.1, g_{\rho}=4$

tive all up to $m_{\rho} \sim 3 \mathrm{TeV}$. A combination of ATLAS and CMS measurements with $\mathcal{L}=3000 \mathrm{fb}^{-1}$ can hence allow sensitivity to compositeness scales up to $m_{\rho} \sim 3-4 \mathrm{TeV}$.

The $95 \%$ CL sensitivity expected for the combination of the three channels for the $S O(5) / S O(4)$ model considered here with $m_{\rho} \sim g_{\rho} f$ on the $\left(\xi, m_{\rho}\right)$ plane is presented in Fig. 7 for three different luminosity scenarios. The nonperturbative limit $g_{\rho}=4 \pi$, where $g_{\rho}$ the physical coupling of the three $\rho$ resonances, is also shown.

It is worth noting that this result might be modified if the impact of fermion resonances is taken into account. This effect is highly model-dependent and relevant only if the $\rho$ resonances interact directly with fermion resonances. In this case the interactions of vector mesons with third generation quarks can be enhanced due to partial compositeness. This does not affect the $\rho$ production cross sections, but modifies the $\rho$ decay widths into third generation quarks. Moreover, if the fermion resonances are light (which is motivated by naturalness arguments) the decays of the vector meson into a SM fermion and a fermion resonance, and the decays of $\rho$ into two fermion resonances, might become kinematically available. This would make the impact on the width and branching ratios of the vector meson even stronger.

\section{Summary and conclusions}

In this paper the compositeness discovery potential for the upcoming LHC runs was presented. A method was proposed to directly probe the Higgs boson compositeness by identifying boosted Higgs events from $\rho \rightarrow V H$ decays. We have demonstrated that a search for compositeness is feasible with a data sample as small as $\mathcal{L}=300 \mathrm{fb}^{-1}$ collected at $\sqrt{s}=13$

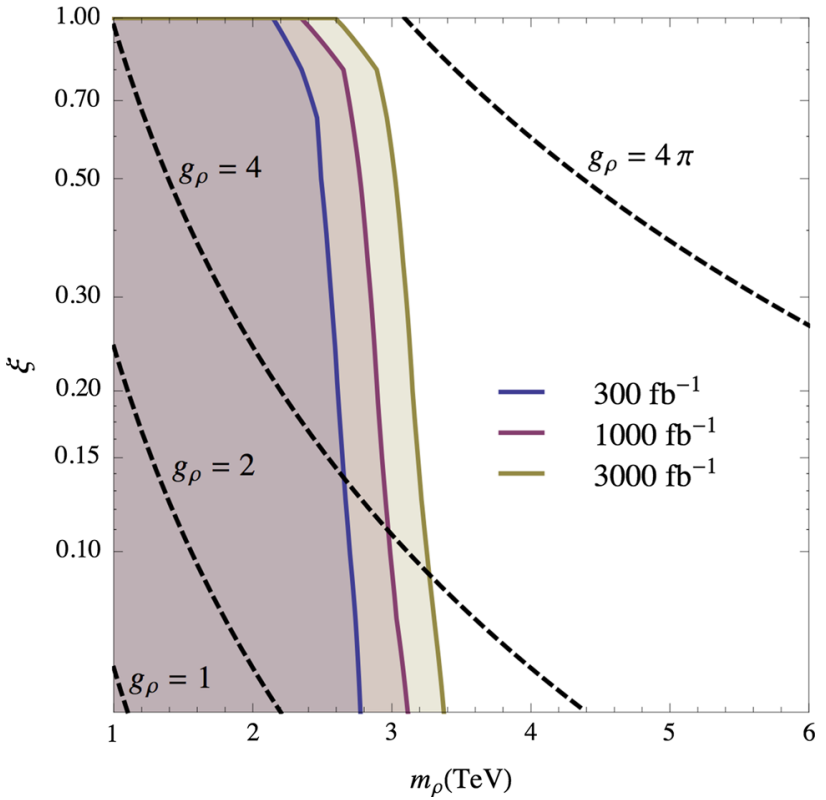

Fig. 7 Summary of the $95 \%$ CL expected sensitivity for a single LHC experiment at $\sqrt{s}=13 \mathrm{TeV}$. The bands show the regions on the $\left(\xi, m_{\rho}\right)$ plane that can be excluded using the combination of the channels $H \rightarrow \gamma \gamma, H \rightarrow Z Z^{*} \rightarrow 4 \ell$, and $H \rightarrow b \bar{b}$. The latter was obtained with an optimistic extrapolation of the results obtained with the $H \rightarrow \gamma \gamma$ channel with the results reported in $[21,22]$. The $g_{\rho}=4 \pi$ line defines the perturbativity boundary above which the effective lagrangian description breaks down

$\mathrm{TeV}$, which corresponds to the data sample recorded by the LHC in the year 2020.

The main result of this work is that compositeness scales up to $g_{\rho} f \sim 3-4 \mathrm{TeV}$ can be probed at the LHC with $\mathcal{L}=$ $3000 \mathrm{fb}^{-1}$ by alone using the VH decay mode. Combining the $\mathrm{VH}$ channel with dilepton and dijet searches could further increase the sensitivity and reach of the search. The proposed search can immediately be employed in the Higgs analyses currently performed by the ATLAS and CMS experiments.

Acknowledgments AK would like to thank the Mainz Institute for Theoretical Physics (MITP) for its hospitality and support during the completion of this work.

Open Access This article is distributed under the terms of the Creative Commons Attribution License which permits any use, distribution, and reproduction in any medium, provided the original author(s) and the source are credited.

Funded by SCOAP $^{3}$ / License Version CC BY 4.0.

\section{Appendix: Effective Lagrangian for a $\rho$ resonance}

Following [11], the PNG bosons $\Pi(x)=\Pi^{\hat{a}}(x) T^{\hat{a}}$ of $\mathcal{G} \rightarrow$ $\mathcal{H}$ symmetry breaking can be described by $U(\Pi)=e^{i \Pi(x) / f}$ transforming as

$$
U(\Pi) \rightarrow g U(\Pi) h^{\dagger}(\Pi, g), \quad g \in \mathcal{G}, h \in \mathcal{H} .
$$


The leading-order effective Lagrangian term describing selfinteractions of these bosons takes the form

$\mathcal{L}^{\Pi}=\frac{f^{2}}{4} \operatorname{Tr}\left\{d_{\mu} d^{\mu}\right\}$

where $d_{\mu}$ is defined by

$$
-i U^{\dagger} D_{\mu} U=d_{\mu}^{\hat{a}} T^{\hat{a}}+E_{\mu}^{a} T^{a}=d^{a}+E^{a}
$$

and $T^{\hat{a}}, T^{a}$ are the broken and unbroken generators of $\mathcal{G}$. The covariant derivative takes into account the external gauging and introduces interactions of PNG bosons with electroweak bosons. For the description of the vector meson transforming as

$$
\left(T^{a} \rho_{\mu}^{a}\right) \rightarrow h\left(T^{a} \rho_{\mu}^{a}\right) h^{\dagger}-\frac{i}{g_{\rho}} h \partial_{\mu} h^{\dagger}, \quad h \in \mathcal{H},
$$

(where in our case $T^{a}$ are $S U(2)_{L}$ generators), we use the general leading-order effective Lagrangian

$\mathcal{L}^{\rho}=-\frac{1}{4 g_{\rho}^{2}} \rho_{\mu \nu}^{a} \rho^{a \mu \nu}+\frac{m_{\rho}^{2}}{2 g_{\rho}^{2}}\left(\rho_{\mu}^{a}-E_{\mu}^{a}\right)^{2}$.

The connection term $E_{\mu}^{a}$ introduces interactions of $\rho$ mesons with PNG bosons and electroweak bosons.

\section{References}

1. F. Englert, R. Brout, Broken symmetry and the mass of gauge vector mesons. Phys. Rev. Lett. 13, 321 (1964)

2. P.W. Higgs, Broken symmetries and the masses of gauge bosons. Phys. Rev. Lett. 13, 508 (1964)

3. G. Guralnik, C. Hagen, T. Kibble, Global conservation laws and massless particles. Phys. Rev. Lett. 13, 585 (1964)

4. C.M.S. Collaboration, Observation of a new boson at a mass of 125 $\mathrm{GeV}$ with the CMS experiment at the LHC. Phys. Lett. B 716, 30 (2012)

5. ATLAS Collaboration, Observation of a new particle in the search for the standard model Higgs boson with the ATLAS detector at the LHC. Phys. Lett. B 716, 1 (2012)

6. CMS Collaboration, Search for narrow resonances in dilepton mass spectra in $p p$ collisions at $\sqrt{s}=7 \mathrm{TeV}$. arXiv:1206.1849 [hep-ex] (2013)

7. ATLAS Collaboration, Search for high-mass dilepton resonances in pp collisions at $\sqrt{s}=8 \mathrm{TeV}$ with the ATLAS detector. arXiv:1405.4123 [hep-ex] (2014)

8. R. Contino, The Higgs as a composite Nambu-Goldstone boson. arXiv: 1005.4269 [hep-ph] (2010)

9. S.R. Coleman, J. Wess, B. Zumino, Structure of phenomenological Lagrangians. 1. Phys. Rev. 177, 2239 (1969)

10. C.G. Callan Jr, S.R. Coleman, J. Wess, B. Zumino, Structure of phenomenological Lagrangians. 2. Phys. Rev. 177, 2247 (1969)
11. R. Contino, D. Marzocca, D. Pappadopulo, R. Rattazzi, On the effect of resonances in composite Higgs phenomenology. JHEP 1110, 081 (2011)

12. M. Bando, T. Kugo, K. Yamawaki, Nonlinear realization and hidden local symmetries. Phys. Rept. 164, 217 (1988)

13. G. Panico, A. Wulzer, The discrete composite Higgs model. JHEP 1109, 135 (2011)

14. R. Contino, C. Grojean, D. Pappadopulo, R. Rattazzi, A. Thamm, Strong Higgs interactions at a linear collider. JHEP 1402, 006 (2014)

15. LHC Higgs Cross-Section Working Group, Handbook of LHC Higgs Cross Sections: 3. Higgs Properties. arXiv:1307.1347 [hepph] (2013)

16. A. Azatov, R. Contino, A. Di Iura, J. Galloway, New prospects for Higgs compositeness in $h \rightarrow Z \gamma$. Phys. Rev. D 88, 075019 (2013)

17. ATLAS Collaboration, Constraints on new phenomena via Higgs boson coupling measurements with the ATLAS detector, ATLASCONF-2014-10 (2014)

18. A. Azatov, J. Galloway, Electroweak symmetry breaking and the Higgs boson: confronting theories at colliders. Int. J. Mod. Phys. A 28, 1330004 (2013)

19. ATLAS Collaboration, Measurements of Higgs boson production and couplings in the four-lepton channel in pp collisions at center-of-mass energies of 7 and $8 \mathrm{TeV}$ with the ATLAS detector. arXiv:1408.5191 [hep-ph] (2014)

20. CMS Collaboration, $\mathrm{H}$ to ZZ to 41, CMS-PAS-FTR-13-003 (2013)

21. ATLAS Collaboration, Search for $H \rightarrow \gamma \gamma$ produced in association with top quarks and constraints on the top quark-Higgs boson Yukawa coupling using data taken at $7 \mathrm{TeV}$ and $8 \mathrm{TeV}$ with the ATLAS detector, ATLAS-CONF-2014-043 (2014)

22. ATLAS Collaboration, Search for the standard model Higgs boson produced in association with top quarks and decaying to $b \bar{b}$ in $p p$ collisions at $\sqrt{s}=8 \mathrm{TeV}$ with the ATLAS detector at the LHC, ATLAS-CONF-2014-011 (2014)

23. J. Alwall et al. Madgraph 5 : Going beyond. arXiv:1106.0522v1 [hep-ph] (2011)

24. T. Sjostrand, S. Mrenna, P. Skands, A brief introduction to PYTHIA 8.1. Comput. Phys. Commun. 178, 852 (2008)

25. S. Ovyn, X. Rouby, V. Lemaitre, DELPHES, a framework for fast simulation of a generic collider experiment. arXiv:0903.2225 [hep$\mathrm{ph}]$ (2009)

26. G. Cowan, K. Cranmer, E. Gross, O. Vitells, Asymptotic formulae for likelihood-based tests of new physics. Eur. Phys. J. C 71, 1-19 (2011)

27. ECFA high luminosity LHC experiments workshop: physics and technology challenges. 94th Plenary ECFA meeting (2013)

28. J. Beringer et al., Review of particle physics (RPP). Phys. Rev. D 86, 010001 (2012)

29. ATLAS Collaboration, Measurement of Higgs boson production in the diphoton decay channel in $p p$ collisions at center-of-mass energies of 7 and $8 \mathrm{TeV}$ with the ATLAS detector. arXiv:1408.7084 [hep-ex] (2014)

30. ATLAS Collaboration, Projections for measurements of Higgs boson cross sections, branching ratios and coupling parameters with the ATLAS detector at a HL-LHC, ATL-PHYS-PUB-2013014 (2013) 